\title{
Identification of potential diagnostic and prognostic biomarkers in non-small cell lung cancer based on microarray data
}

\author{
RU HUANG ${ }^{1}$ and LEI GAO ${ }^{1,2}$ \\ ${ }^{1}$ Department of Heart Failure, Research Center for Translational Medicine, Shanghai East Hospital, \\ Tongji University School of Medicine; ${ }^{2}$ Key Laboratory of Arrhythmias of The Ministry of Education of China, \\ Research Institute of Heart Failure, Shanghai East Hospital, Dalian Medical University, Shanghai 200120, P.R. China
}

Received December 21, 2016; Accepted February 1, 2018

DOI: $10.3892 / \mathrm{ol} .2018 .8153$

\begin{abstract}
Non-small cell lung cancer (NSCLC) is the most commonly diagnosed subtype of lung cancer, and the leading cause of cancer-associated mortalities worldwide. However, NSCLC is typically diagnosed at a late stage of disease due to a lack of effective diagnostic methods. In the present study, the GSE19804 dataset was obtained from the Gene Expression Omnibus, and a number of differentially expressed genes were identified between NSCLC and adjacent normal tissues. Based on functional and pathway enrichment analyses, five hub genes (cell-division cycle 20, centromere protein F, kinesin family member 2C, BUB1 mitotic checkpoint serine/threonine kinase and ZW10 interacting kinetochore protein) were selected. After verifying that the mRNA level of these hub genes was also upregulated in NSCLC tissues by using the GSE10072 dataset and in cell lines by reverse transcription-quantitative polymerase chain reaction. The diagnostic and prognostic potentials of these five gene candidates were evaluated using receiver operating characteristic curves and survival analyses. Taken together, the present study identified five candidates that are overexpressed in NSCLC tissues and could also serve as potential diagnostic and prognostic biomarkers for patients with NSCLC.
\end{abstract}

\section{Introduction}

Lung cancer is the leading cause of cancer-associated mortalities worldwide, with $\sim 1.6$ million new cases each year (1). Non-small cell lung cancer (NSCLC) accounts for $~ 85 \%$ of all lung cancer cases, which consists of three major histological subtypes, including adenocarcinoma, squamous cell carcinoma

Correspondence to: Dr Lei Gao, Department of Heart Failure, Research Center for Translational Medicine, Shanghai East Hospital, Tongji University School of Medicine, 150 Jimo Road, Shanghai 200120, P.R. China

E-mail: gldlykdx@163.com

Key words: bioinformatics analysis, non-small cell lung cancer, microarray data, differentially expressed genes, biomarkers and large cell carcinoma (2). Due to a lack of methods for detection of NSCLC that are suitable for the general population, NSCLC is typically diagnosed at late stages of the disease where metastasis is present (3). Therefore, it is of great importance to elucidate the molecular mechanisms underlying the pathogenesis of NSCLC and identify effective biomarkers for early diagnosis and prognosis.

Microarray technology is a high-throughput platform used to analysis gene expression and has been broadly used to obtain gene alteration during tumorigenesis and identify prognostic biomarkers in patients with cancer (4-6). However, genes identified by one cohort may be difficult to be confirmed in other cohorts (7). Therefore, in order to address this problem, it is necessary to validate genes in several individual studies.

In the present study, the aim was to identify the potential genes that serve as diagnostic and prognostic biomarkers for patients with NSCLC through retrieving the microarray data from public databases and comprehensive bioinformatics analysis. Gene expression profiles between tumor and adjacent normal tissues were illustrated, and differentially expressed genes (DEGs) based on the GSE19804 dataset were identified. By combining functional pathway and protein-protein interaction (PPI) analyses, five hub genes were selected, including cell division cycle $20(C D C 20)$, centromere protein $\mathrm{F}(C E N P F)$, kinesin family member $2 \mathrm{C}(K I F 2 C)$, BUB1 mitotic checkpoint serine/threonine kinase $(B U B 1)$ and ZW10 interacting kinetochore protein (ZWINT). Furthermore, the levels of mRNA expression of these genes were validated using an additional dataset (GSE10072) and NSCLC cell lines. Receiver operating characteristic (ROC) curves and survival analyses were employed to evaluate the diagnostic and prognostic potentials of five hub genes.

\section{Materials and methods}

Microarray data. The gene expression profiles of GSE19804 and GSE10072 were obtained from the Gene Expression Omnibus (GEO, http://www.ncbi.nlm.nih.gov/geo). These two gene expression datasets were analyzed using the Affymetrix platform (Affymetrix Human Genome U133 Plus 2.0 Array; Thermo Fisher Scientific, Inc., Waltham, MA, USA). The GSE19804 gene expression profile submitted by Lu et al (8) included 60 pairs of clinical NSCLC samples, which consisted 
of 56 adenocarcinoma, 3 bronchioloalveolar carcinoma, and 1 squamous carcinoma, and corresponding adjacent normal tissue samples. The GSE10072 gene expression profile consisted of 58 adenocarcinoma samples (16 non-smokers, 18 former smokers and 24 current smokers) and 49 non-tumor samples (15 non-smokers, 18 former smokers and 16 current smokers) (9).

Processing of data. Raw microarray data files of the two datasets were downloaded from the GEO database. GEO2R (http://www.ncbi.nlm.nih.gov/geo/geo2r/), an online tool that compares two or more groups of samples in the same experimental setting, was used to analyze the raw data (10). False Discovery Rate (FDR) adjusted P-value of 0.05 and $\mid \log F C l>1$ were set as the cut-off criteria.

Functional and pathway enrichment analyses of DEGs. Gene ontology (GO) analysis was processed by the Database for Annotation, Visualization and Integrated Discovery (DAVID) (http://david.abcc.ncifcrf.gov/) to elucidate the biological function of genes in NSCLC. Kyoto Encyclopedia of Genes and Genomes (KEGG) pathway enrichment analysis was performed to identify DEGs using the DAVID database. $\mathrm{P}<0.05$ was set as the threshold.

Construction of PPI network and module analysis. The functional interaction of proteins can shed light on the molecular mechanism underlying NSCLC. The online database STRING (version 10.0, http://string.embl.de/) can be used in the evaluation of PPIs (11). The STRING database includes 9,643,763 proteins from 2,031 organisms. In order to evaluate the PPIs among the DEGs, DEGs were mapped to the STRING database. A confidence score $>0.7$ was selected as significant. In addition, the degree of the nodes in PPI network was calculated, and the nodes with a higher degree were selected as hub proteins. Furthermore, Cytoscape software (version 3.4.0, http://cytoscape.org/) was employed to construct PPI networks. The plug-in Molecular Complex Detection (MCODE) was performed to screen modules of the PPI network with the threshold set as follows: MCODE scores $>10$. The GO and KEGG analysis of genes in the module was performed using the DAVID online tool as aforementioned.

Cell culture. The cell lines, human bronchial epithelial (HBE1), A549 and H322, were gifted from Professor Zeyao Tang (Dalian Medical University, Dalian, China) (12). The cells were maintained in high-glucose Dulbecco's modified Eagles medium (Gibco; Thermo Fisher Scientific, Inc.) supplemented with $10 \%$ fetal bovine serum (GE Healthcare Life Sciences, Logan, UT, USA), $100 \mathrm{U} / \mathrm{ml}$ penicillin and $100 \mu \mathrm{g} / \mathrm{ml}$ streptomycin (Gibco; Thermo Fisher Scientific, Inc.). The cells were incubated at $37^{\circ} \mathrm{C}$ in a humidified chamber with $5 \% \mathrm{CO}_{2}$.

Reverse transcription-quantitative polymerase chain reaction (RT-qPCR). Total RNA from cells lines, including HBE1, A549, and H322, were extracted by using the TRIzol ${ }^{\circledR}$ regent (Invitrogen; Thermo Fisher Scientific, Inc.). The cDNA of mRNA was synthesized using the PrimeScript ${ }^{\mathrm{TM}}$ RT reagent kit (Takara Bio Inc., Otsu, Japan). RT-qPCR was carried out using the 7500 Real-time PCR system (Thermo Fisher
Scientific, Inc.) at $95^{\circ} \mathrm{C}$ for initial denaturation for $10 \mathrm{~min}$, followed by 40 cycles at $95^{\circ} \mathrm{C}$ for $15 \mathrm{sec}$, and $60^{\circ} \mathrm{C}$ for $1 \mathrm{~min}$ with the SYBR ${ }^{\circledR}$ Green mix (Takara Bio Inc., Japan). Data were analyzed by using the comparative $\mathrm{Cq}(\Delta \Delta \mathrm{Cq})$ to determine the relative gene expression, and GAPDH was used as an endogenous control (13). The primers were synthesized by Shanghai GenePharma Co., Ltd., (Shanghai, China). The following primer pairs was used to measure the amount of GAPDH: Forward, 5'-GGAGCGAGATCCCTCCAAAAT-3' and reverse, 5'-GGCTGTTGTCATACTTCTCATGG-3'.

ROC analysis. ROC curve analysis was performed using the MedCalc software packages (version 16.8.4; MedCalc Software bvba, Ostend, Belgium). The area under the curve (AUC) values with $95 \%$ confidence interval (CI) were calculated to evaluate the overall performance of the diagnostic tests.

Survival analysis of hub genes. Kaplan-Meier plotter (www. kmplot.com), an online survival analysis tool, was used to evaluate the prognostic value of biomarkers of breast, ovarian, lung and gastric cancer (14). Patients with NSCLC were divided into high and low expression groups using the median level, which was included in the low expression group, as the cutoff value. To analyze the association between gene expression and clinical outcomes, Kaplan-Meier plots was employed to compare the overall survival ratio between the two groups, and the log rank P-value and hazard ratio (HR) with $95 \%$ confidence intervals $(\mathrm{CI})$ were calculated and displayed.

Statistical analysis. The data are expressed as the mean \pm standard deviation of three replicates. Statistical differences were assessed using one-way analysis of variance test and Tukey's multiple comparisons test. SPSS software (version 17.0; SPSS, Inc., Chicago, IL, USA) was used to analyze the data. $\mathrm{P}<0.05$ was considered to indicate a statistically significant difference.

\section{Results}

Identification of DEGs. By using the threshold (adjusted P-vale of 0.05 and fold change $>2$ ), a total of 1,412 DEGs were identified in the GSE19804 dataset. Among these genes, 453 genes were upregulated, and 959 genes were downregulated. A heat-map illustrating the expression of the top 50 up and downregulated DEGs is shown in Fig. 1.

Analysis of GO terms and KEGG pathway enrichment. To further elucidate the functions of the identified DEGs in NSCLC, GO and KEGG pathway enrichment analyses were employed. As shown in Table I, GO analysis of upregulated DEGs in NSCLC indicated that these genes were associated with 'mitotic cell cycle', 'mitotic nuclear division' and the 'cell cycle process'. KEGG pathway enrichment analysis of DEGs revealed that upregulated DEGs were largely enriched in cell cycle and extracellular matrix (ECM)-receptor interaction pathways, while downregulated DEGs were enriched in 'malaria' and 'tumor necrosis factor (TNF) signaling pathways' (Table I). These results suggest that upregulated DEGs in NSCLC may be largely involved in the progression of the cell cycle. 


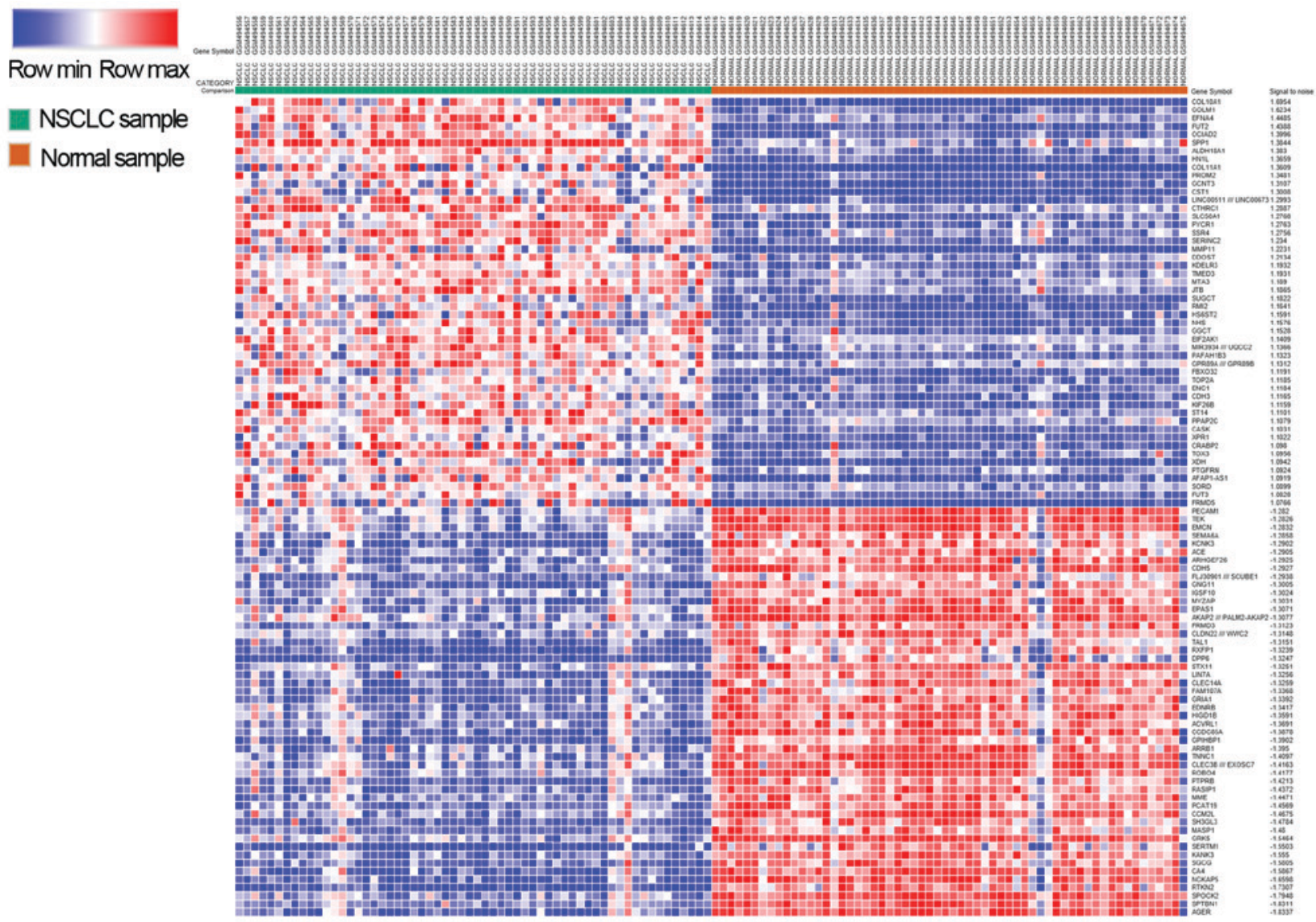

Figure 1. Heat map of the top 100 differentially expressed genes, including 50 up- and downregulated genes. Red, upregulation; blue, downregulation. NSCLC, non-small cell lung cancer.

Construction of PPI network and selection of modules. Based on the analysis of DEGs in the STRING database, a PPI network of DEGs containing 1,291 nodes and 2,854 edges was constructed. By using the plug-in MCODE in Cytoscape, the top 3 modules in the PPI network was obtained (Fig. 2A-C), and KEGG analysis of genes in the corresponding modules was also performed (Fig. 2D-F). Consistent with the KEGG analysis of DEGs, function enrichment analysis of genes in the top 3 modules indicated that these hub genes were also enriched in 'cell cycle progression' (Fig. 2E). Therefore, the present study focused on the 5 hub genes associated with cell cycle progression including $C D C 20, C E N P F, K I F 2 C, B U B 1$ and ZWINT.

Validation of 5 selected hub genes. Although 5 hub genes were selected by KEGG analysis the genes in these 3 modules, these 5 selected genes may be limited to the diagnosis or prognosis for non-smoking female patients with NSCLC. In order to elucidate whether these genes can be non-selectively applied to patients with NSCLC, as previously reported $(4,15)$, an additional dataset and RT-qPCR were employed to validate the mRNA level of these genes in NSCLC samples and cell lines. Since the GSE19804 dataset included 56 non-smoking female adenocarcinoma samples (8), the present study searched for a dataset that included adenocarcinoma and simultaneously excluded the effects of sex and smoking. Based on the aforementioned criterion, the GSE10072 database was identified as suitable. Using the GSE10072 dataset, it was detected that the mRNA level of these 5 genes were also overexpressed in NSCLC samples (Fig. 3A-E). In addition, the RT-qPCR results also validated that the mRNA level of these genes were overexpressed in NSCLC cell lines including A549 and H322 (16), when compared with the control cell line HBE1 (Fig. 3F). H322 may be identical to another uncommonly used NSCLC cell line H322M (https://web.expasy.org/cellosaurus/CVCL_1556). Taken together, these results suggest that these 5 hub genes may be novel gene signatures for patients with NSCLC.

ROC analysis of selected hub genes. To evaluate the diagnostic value of these 5 hub genes, ROC analysis was conducted based on these 2 datasets. The present study demonstrated that the sensitivity and specificity of these 5 genes was relatively high. As shown in Fig. 4A, the AUC values for $C D C 20, C E N P F$, KIF 2C, BUB1 and ZWINT were $0.927,0.906,0.887,0.876$, and 0.937 , respectively in the GSE19804 dataset, while the values were $0.958,0.944,0.923,0.897$, and 0.942 , respectively in the GSE10072 dataset (Fig. 4B). These results indicate that these 5 hub genes may be sensitive and specific in distinguishing NSCLC tissues from normal tissues.

Kaplan-Meier plotter analysis of selected hub genes. The prognostic value of these 5 genes in PPI network was evaluated using the Kaplan-Meier plotter as previous described (14). Based on the low and high expression of each hub gene, the overall survival of patients with NSCLC was obtained for each gene. As shown in Fig. 5, the high mRNA expression of 
Table I. Functional and pathway enrichment analysis of upregulated and downregulated genes in non-small cell lung cancer.

\begin{tabular}{|c|c|c|c|}
\hline Category & Term/gene function & Gene count & P-value \\
\hline \multicolumn{4}{|l|}{ Upregulated } \\
\hline \multicolumn{4}{|l|}{$\mathrm{GO}$} \\
\hline 0000278 & Mitotic cell cycle & 62 & $1.1 \times 10^{14}$ \\
\hline 0007067 & Mitotic nuclear division & 39 & $1.2 \times 10^{12}$ \\
\hline 0022402 & Cell cycle process & 75 & $1.9 \times 10^{12}$ \\
\hline 005130 & Cell division & 45 & $2.2 \times 10^{12}$ \\
\hline 0044772 & Mitotic cell cycle phase transition & 42 & $3.2 \times 10^{12}$ \\
\hline 0000793 & Condensed chromosome & 22 & $7.9 \times 10^{8}$ \\
\hline 0005578 & Proteinaceous extracellular matrix & 27 & $2.1 \times 10^{6}$ \\
\hline 0005819 & Spindle & 24 & $2.3 \times 10^{6}$ \\
\hline 0000776 & Kinetochore & 15 & $5.4 \times 10^{6}$ \\
\hline \multicolumn{4}{|l|}{ KEGG } \\
\hline hsa04110 & Cell cycle & 19 & $1.0 \times 10^{9}$ \\
\hline hsa04512 & ECM-receptor interaction & 14 & $1.7 \times 10^{7}$ \\
\hline hsa04115 & P53 signaling pathway & 12 & $6.0 \times 10^{7}$ \\
\hline hsa04974 & Protein digestion and absorption & 11 & $5.6 \times 10^{5}$ \\
\hline hsa04510 & Focal adhesion & 15 & $5.5 \times 10^{4}$ \\
\hline \multicolumn{4}{|c|}{ Downregulated } \\
\hline hsa05144 & Malaria & 15 & $2.2 \times 10^{7}$ \\
\hline hsa04668 & TNF signaling pathway & 19 & $1.6 \times 10^{5}$ \\
\hline hsa04530 & Tight junction & 21 & $5.4 \times 10^{5}$ \\
\hline hsa04514 & Chemokine signaling pathway & 23 & $5.4 \times 10^{4}$ \\
\hline hsa04360 & Axon guidance & 17 & $1.6 \times 10^{3}$ \\
\hline
\end{tabular}

GO, gene ontology; KEGG, Kyoto Encyclopedia of Genes and Genomes; ECM, extracellular matrix; TNF, tumor necrosis factor.

\begin{tabular}{|c|c|c|c|c|}
\hline \multirow{7}{*}{ 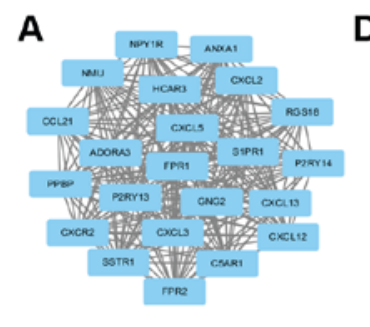 } & \multicolumn{2}{|l|}{ Dene set } & $P$ value & Nodes \\
\hline & \multirow{2}{*}{\multicolumn{2}{|c|}{ Chemokine signaling pathway }} & \multirow[t]{2}{*}{$3.02 \times 10^{11}$} & CCL21, CXCL12, CXCL13, CXCL2, \\
\hline & & & & CXCL3, CXCL5, CXCR2, GNG2, PPBP \\
\hline & \multirow{2}{*}{\multicolumn{2}{|c|}{ Neuroactive ligand-receptor interaction }} & \multirow[t]{2}{*}{$6.32 \times 10^{10}$} & ADORA3, C5AR1, FPR1, FPR2, NPY1R, \\
\hline & & & & P2RY13, P2RY14, S1PR1, SSTR1 \\
\hline & \multirow{2}{*}{\multicolumn{2}{|c|}{ Cytokine-cytokine receptor interaction }} & \multirow[t]{2}{*}{$1.6 \times 10^{8}$} & CCL2s1, CXCL12, CXCL13, CXCL2, \\
\hline & & & & CXCL3, CXCL5, CXCR2, PPBP \\
\hline \multirow{5}{*}{ B $\quad$ E } & \\
\hline & \multicolumn{2}{|l|}{ Gene set } & $P$ value & Nodes \\
\hline & \multicolumn{2}{|l|}{ cGMP-PKG signaling pathway } & $1.68 \times 10^{4}$ & ADRA1A, AGTR1, EDNRB, PLCB4, RGS2 \\
\hline & \multicolumn{2}{|c|}{ Neuroactive ligand-receptor interaction } & $1.68 \times 10^{4}$ & ADRA1A, AGTR1, EDNRB, F2RL1, KISS1R, P2RY1 \\
\hline & \multicolumn{2}{|l|}{ Cell cycle } & $1.28 \times 10^{3}$ & $B \cup B 1, B \cup B 1 B, C D C 20, M A D 2 L 1$ \\
\hline \multirow[t]{4}{*}{ C } & \multicolumn{2}{|l|}{ Gene set } & Nodes & \\
\hline & ECM-receptor interaction & $3.18 \times 10^{19}$ & \multicolumn{2}{|c|}{ COL11A1, COL1A1, COL1A2, COL3A1, COL4A3, COL5A1, } \\
\hline & Focal adhesion & $2.71 \times 10^{17}$ & \multicolumn{2}{|c|}{ COL11A1, COL1A1, COL1A2, COL3A1, COL4A3, COL5A1, } \\
\hline & PI3K-Akt signaling pathway & $7.81 \times 10^{15}$ & \multicolumn{2}{|c|}{$\begin{array}{l}\text { COL11A1, COL1A1, COL1A2, COL3A1, COL4A3, COL5A1, } \\
\text { COL5A2, EGF, ITGA1, ITGA11, ITGA8, VWF }\end{array}$} \\
\hline
\end{tabular}

Figure 2. Top 3 modules obtained from the protein-protein interaction network of differentially expressed genes. (A) Module 1 and (B) its enriched KEGG pathways. (C) Module 2 and (D) its enriched KEGG pathways. (E) Module 3 and (F) its enriched KEGG pathways. KEGG, Kyoto Encyclopedia of Genes and Genomes. 

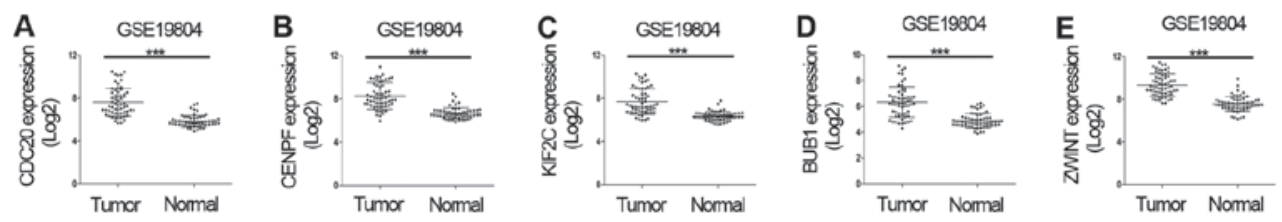

$\mathbf{F}$
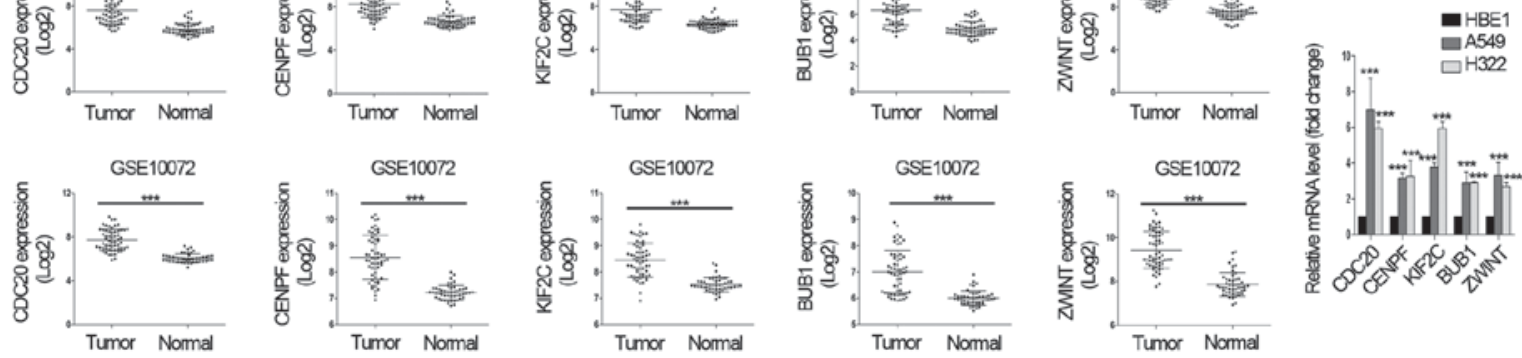

Figure 3. Validation of 5 selected differentially expressed genes. The mRNA level of 5 hub genes, including (A) CDC20, (B) CENPF, (C) KIF2C, (D) BUBI and (E) ZWINT in two datasets (GSE19804 and GSE10072). (F) The levels of CDC20, CENPF, KIF2C, BUB1 and ZWINT mRNA were validated by reverse transcription-quantitative polymerase chain reaction. Total RNA was isolated from cultured non-small cell lung cancer cell lines (A549, H322 and control cell line HBE1). The values are expressed as the mean \pm standard deviation of three replicates. ${ }^{* * *} \mathrm{P}<0.01$. CDC20, cell division cycle 20 ; CENPF, centromere protein F; KIF2C, kinesin family member 2C; BUB1, BUB1 mitotic checkpoint serine/threonine kinase; ZWINT, ZW10 interacting kinetochore protein.
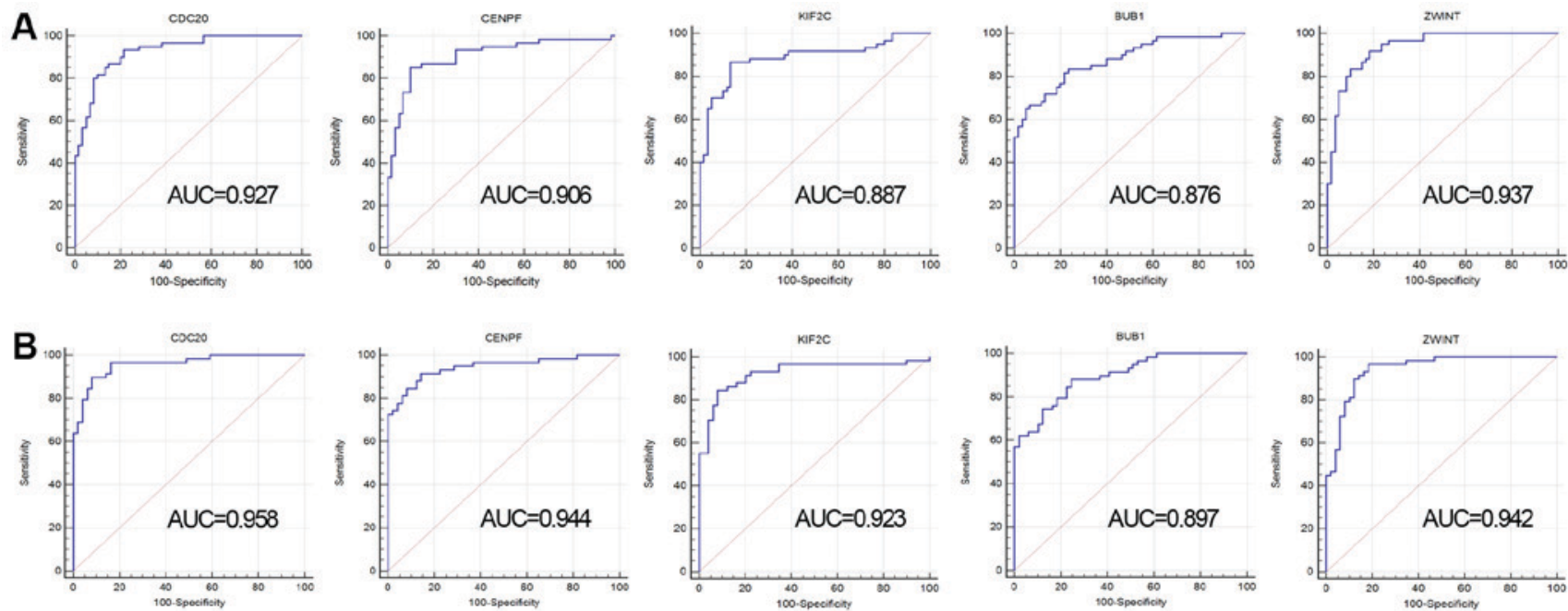

Figure 4. Diagnostic value of $C D C 20, C E N P F, K I F 2 C, B U B 1$ and ZWINT in NSCLC. (A) Receiver operating characteristic analysis of 5 hub genes based on the GSE19804 dataset. (B) Receiver operating characteristic analysis of 5 hub genes in the GSE10072 dataset. AUC, area under the curve; CDC20, cell division cycle 20; CENPF, centromere protein F; KIF2C, kinesin family member 2C; BUB1, BUB1 mitotic checkpoint serine/threonine kinase; ZWINT, ZW10 interacting kinetochore protein.

CDC20 (HR, 1.82; CI, 1.6-2.07) was associated with a poorer overall survival for patients with NSCLC. Similar associations were detected for: CENPF (HR, 1.57, CI, 1.38-1.78), KIF2C (HR, 1.78; CI, 1.57-2.03), BUB1 (HR, 1.83; CI, 1.61-2.09) and ZWINT (HR, 1.5; CI, 1.32-1.71). These results indicate that these 5 hub genes may serve as potential prognostic biomarkers for patients with NSCLC.

\section{Discussion}

In the present study, the mRNA level of five genes identified from the GSE19804 dataset, CDC20, CENPF, KIF2C, BUBI and ZWINT, were demonstrated to be upregulated in NSCLC samples. This was validated using the GSE10072 dataset and RT-qPCR. By employing ROC curve and Kaplan-Meier plotter analyses, it was further demonstrated that these five candidates were sensitive and specific in distinguishing NSCLC tissues from normal tissues, and these candidate genes were associated with a poor overall survival in patients with NSCLC.
Although advances in surgery and chemotherapy have improved the prognosis of patients with NSCLC, NSCLC remains the leading cause of cancer-associated mortalities worldwide (1). However, due to a lack of specific biomarkers and typical symptoms, patients with NSCLC are commonly diagnosed at late stages of the disease (3). Therefore, it is important to elucidate the molecular mechanisms of NSCLC and identify more specific biomarkers for patients with NSCLC.

A combination of high-throughput sequencing and bioinformatics analysis has been previously employed to search for sensitive biomarkers for patients with $\operatorname{NSCLC}(4,8)$. In the present study, in order to identify potential biomarkers of NSCLC, the gene expression profiles in the dataset GSE19804 were obtained and DEGs in NSCLC tissues were subsequently identified (Fig. 1). Using a combination of PPI analysis and subsequent selection of modules, five hub genes (CDC20, $C E N P F, K I F 2 C, B U B 1$ and ZWINT) that were overexpressed in NSCLC tissues were selected as potential candidates (Fig. 2). However, the repeatability of a study with a single dataset is 

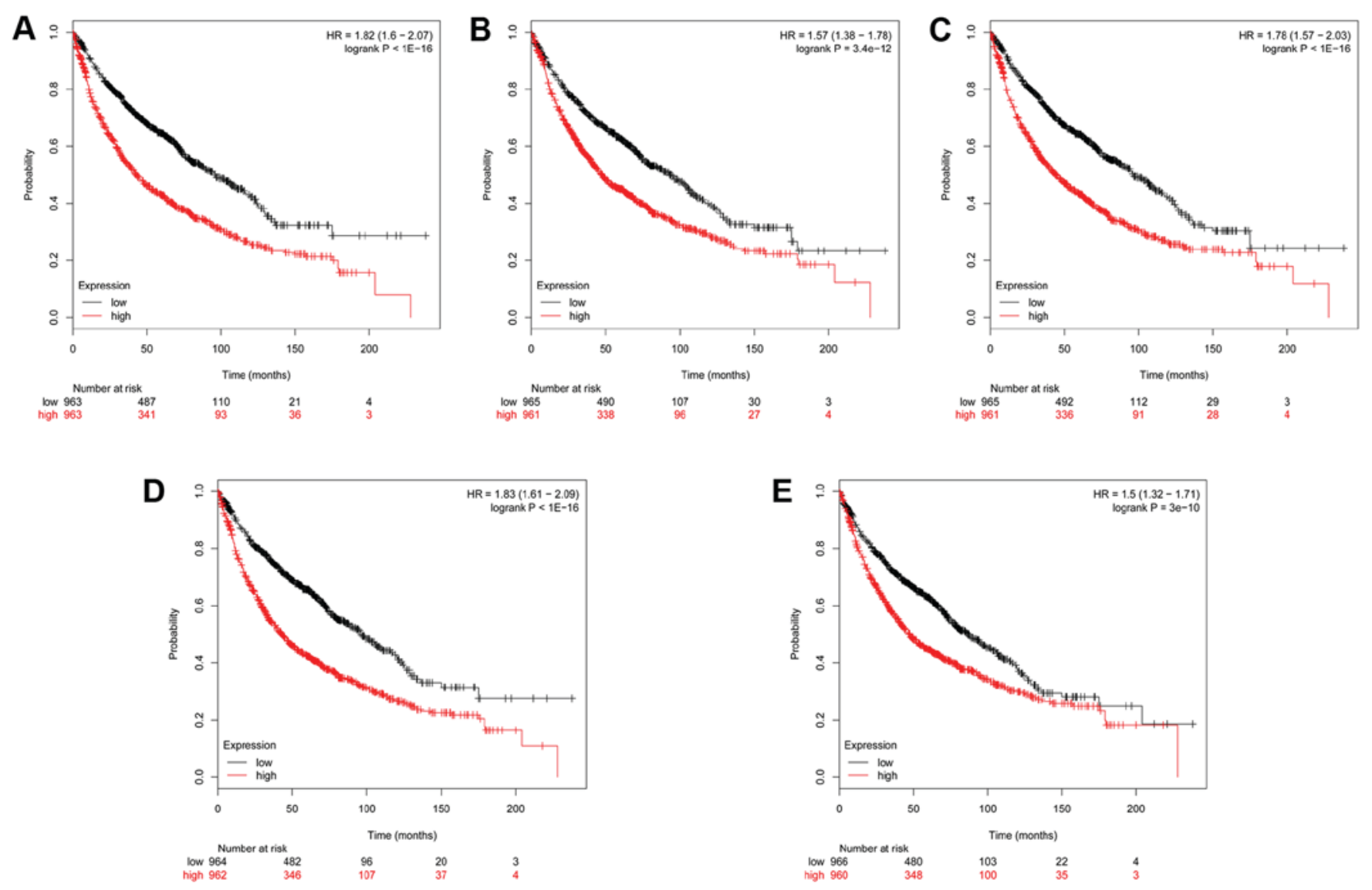

Figure 5. Kaplan-Meier survival analysis of 5 hub genes in patients with non-small cell lung cancer. Prognostic value of (A) $C D C 20$, (B) $C E N P F$, (C) KIF2C, (D) BUB1 and (E) ZWINT were evaluated using the Kaplan-Meier plotter. The Affymetrix IDs of the genes are as follows: CDC20, 202870_s_; CENPF, 209172_s_; KIF2C, 209408_at; BUB1, 209642_at; ZWINT, 204026_s_. CDC20, cell division cycle 20; CENPF, centromere protein F; HR, hazard ratio; KIF2C, kinesin family member 2C; BUB1, BUB1 mitotic checkpoint serine/threonine kinase; ZWINT, ZW10 interacting kinetochore protein.

usually insufficient. Therefore, an additional two methods were used to validate the overexpression of selected genes in NSCLC tissues and cell lines. The upregulation of the candidate genes were validated by using the GSE10072 dataset and by RT-qPCR (Fig. 3). The 5 candidates were further evaluated using the ROC and Kaplan-Meier plotter analyses to assess their diagnostic and prognostic values. Notably, the results obtained were consistent with those of previous studies $(4,6)$. These identified candidates were also verified to be potential diagnostic and prognostic biomarkers for patients with NSCLC.

The cell cycle is an evolutionarily conserved process that is regulated by several molecules, including cyclins and cyclin-dependent kinases. Cell cycle is critical for the growth and development of mammalian cells (17). Mutations in these proteins and subsequent cell cycle aberrations are common hallmarks of human cancer (18). Although these aberrant cell cycle-associated molecules are not specific to a particular cancer type, they can still serve as potential candidates for the diagnosis or prognosis of patients with cancer $(4,15)$. For instance, the level of cyclinB2, a member of the cyclin family, has been regarded as an unfavorable predictor for the clinical progression and prognosis for patients with NSCLC (19). The present study also identified 5 cell cycle-associated candidates (20-24), which are sensitive and specific in distinguishing NSCLC from normal tissues, and these candidate genes were associated with poor prognosis of NSCLC. Among these candidates, the overexpression of $C D C 20$ has been used to predict the poor prognosis of patients with NSCLC (25). Furthermore, a number of chemotherapeutics have been designed to target these cell cycle-associated molecules and therefore eradicate cancer cells (26). The present study postulates that these 5 identified candidates may possess the potential to serve as novel therapeutic targets for patients with NSCLC.

In summary, the present study has demonstrated that the candidates $C D C 20, C E N P F, K I F 2 C, B U B 1$, and ZWINT are overexpressed in NSCLC tissues, which may be unfavorable prognostic biomarkers for patients with NSCLC. By classifying patients into high- and low-risk groups, patients may benefit from more accurate decision-making in treatment selection and ultimately have an improved clinical outcome. However, due to the different histopathological typing and grouping methods used in the two datasets, further validation of these five candidates for their diagnostic and prognostic values in the clinical samples are required.

\section{Acknowledgments}

Not applicable.

\section{Funding}

No funding was received. 


\section{Availability of data and materials}

The datasets used and/or analyzed during the current study are available from the corresponding author on reasonable request.

\section{Author's contributions}

LG initiated the project and designed the research plan. RH performed the experiments and analyzed the data. RH and LG wrote and approved the manuscript.

\section{Ethics approval and consent to participate}

Not applicable.

\section{Consent for publication}

Not applicable.

\section{Competing interests}

The authors declare that they have no competing interests.

\section{References}

1. Smith RA, Manassaram-Baptiste D, Brooks D, Doroshenk M, Fedewa S, Saslow D, Brawley OW and Wender R: Cancer screening in the United States, 2015: A review of current American cancer society guidelines and current issues in cancer screening. CA Cancer J Clin 65: 30-54, 2015.

2. Thomas A, Liu SV, Subramaniam DS and Giaccone G: Refining the treatment of NSCLC according to histological and molecular subtypes. Nat Rev Clin Oncol 12: 511-526, 2015.

3. van Meerbeeck JP, Fennell DA and De Ruysscher DK: Small-cell lung cancer. Lancet 378: 1741-1755, 2011.

4. Lu Y, Lemon W, Liu PY, Yi Y, Morrison C, Yang P, Sun Z, Szoke J, Gerald WL, Watson M, et al: A gene expression signature predicts survival of patients with stage I non-small cell lung cancer. PLoS Med 3: e467, 2006.

5. Chen F, Xiang CX, Zhou Y, Ao XS, Zhou DQ, Peng P, Zhang HQ, Liu HD and Huang X: Gene expression profile for predicting survival of patients with meningioma. Int J Oncol 46: 791-797, 2015.

6. Xu Z, Zhou Y, Cao Y, Dinh TL, Wan J and Zhao M: Identification of candidate biomarkers and analysis of prognostic values in ovarian cancer by integrated bioinformatics analysis. Med Oncol 33: 130, 2016

7. Ioannidis JP, Allison DB, Ball CA, Coulibaly I, Cui X, Culhane AC, Falchi M, Furlanello C, Game L, Jurman G, et al: Repeatability of published microarray gene expression analyses. Nat Genet 41: 149-155, 2009.

8. Lu TP, Tsai MH, Lee JM, Hsu CP, Chen PC, Lin CW, Shih JY, Yang PC, Hsiao CK, Lai LC and Chuang EY: Identification of a novel biomarker, SEMA5A, for non-small cell lung carcinoma in nonsmoking women. Cancer Epidemiol Biomarkers Prev 19: 2590-2597, 2010.

9. Landi MT, Dracheva T, Rotunno M, Figueroa JD, Liu H, Dasgupta A, Mann FE, Fukuoka J, Hames M, Bergen AW, et al: Gene expression signature of cigarette smoking and its role in lung adenocarcinoma development and survival. PLoS One 3 : e1651, 2008.
10. Barrett T, Wilhite SE, Ledoux P, Evangelista C, Kim IF, Tomashevsky M, Marshall KA, Phillippy KH, Sherman PM, Holko M, et al: NCBI GEO: Archive for functional genomics data sets-update. Nucleic Acids Res 41 (Database Issue): D991-D995, 2013.

11. Szklarczyk D, Franceschini A, Wyder S, Forslund K, Heller D, Huerta-Cepas J, Simonovic M, Roth A, Santos A, Tsafou KP, et al: STRING v10: Protein-protein interaction networks, integrated over the tree of life. Nucl Acids Res 43 (Database Issue): D447-D452, 2015.

12. Sun B, Gao L, Ahsan A, Chu P, Song Y, Li H, Zhang Z, Lin Y, Peng J, Song Z, et al: Anticancer effect of SZC015 on lung cancer cells through ROS-dependent apoptosis and autophagy induction mechanisms in vitro. Int Immunopharmacol 40: 400-409, 2016.

13. Livak KJ and Schmittgen TD: Analysis of relative gene expression data using real-time quantitative PCR and the 2(-Delta Delta C(T)) method. Methods 25: 402-408, 2001.

14. Győrffy B, Surowiak P, Budczies J and Lanczky A: Online survival analysis software to assess the prognostic value of biomarkers using transcriptomic data in non-small-cell lung cancer. PLoS One 8: e82241, 2013.

15. Shi YX, Zhu T, Zou T, Zhuo W, Chen YX, Huang MS, Zheng W, Wang CJ, Li X, Mao XY, et al: Prognostic and predictive values of CDK1 and MAD2L1 in lung adenocarcinoma. Oncotarget 7: 85235-85243, 2016.

16. Gong Y, Yao E, Shen R, Goel A, Arcila M, Teruya-Feldstein J, Zakowski MF, Frankel S, Peifer M, Thomas RK, et al: High expression levels of total IGF-1R and sensitivity of NSCLC cells in vitro to an anti-IGF-1R antibody (R1507). PLoS One 4: e7273, 2009.

17. Dominguez-Brauer C, Thu KL, Mason JM, Blaser H, Bray MR and Mak TW: Targeting mitosis in cancer: Emerging strategies. Mol Cell 60: 524-536, 2015.

18. Hydbring P, Malumbres M and Sicinski P: Non-canonical functions of cell cycle cyclins and cyclin-dependent kinases. Nat Rev Mol Cell Biol 17: 280-292, 2016.

19. Qian X, Song X, He Y, Yang Z, Sun T, Wang J, Zhu G, Xing W and You C: CCNB2 overexpression is a poor prognostic biomarker in Chinese NSCLC patients. Biomed Pharmacother 74: 222-227, 2015.

20. Frescas D and Pagano M: Deregulated proteolysis by the F-box proteins SKP2 and beta-TrCP: Tipping the scales of cancer. Nat Rev Cancer 8: 438-449, 2008.

21. Varis A, Salmela AL and Kallio MJ: Cenp-F (mitosin) is more than a mitotic marker. Chromosoma 115: 288-295, 2006.

22. Wordeman L: Microtubule-depolymerizing kinesins. Curr Opin Cell Biol 17: 82-88, 2005.

23. Taylor SS and McKeon F: Kinetochore localization of murine Bub1 is required for normal mitotic timing and checkpoint response to spindle damage. Cell 89: 727-735, 1997.

24. Woo Seo D, Yeop You S, Chung WJ, Cho DH, Kim JS and Su Oh J: Zwint-1 is required for spindle assembly checkpoint function and kinetochore-microtubule attachment during oocyte meiosis. Sci Rep 5: 15431, 2015.

25. Kato T, Daigo Y, Aragaki M, Ishikawa K, Sato M and Kaji M: Overexpression of CDC20 predicts poor prognosis in primary non-small cell lung cancer patients. J Surg Oncol 106: 423-430, 2012.

26. Malumbres M: Therapeutic opportunities to control tumor cell cycles Clin Transl Oncol 8: 399-408, 2006.

(i) (9) This work is licensed under a Creative Commons Attribution-NonCommercial-NoDerivatives 4.0 International (CC BY-NC-ND 4.0) License. 